Pacific

Journal of

Mathematics

BOUNDARY-CLUSTERED INTERFACES FOR THE ALLEN-CAHN EQUATION

Andrea Malchiodi, WeI-Ming Ni AND Juncheng WeI

Volume $229 \quad$ No. 2

February 2007 


\title{
BOUNDARY-CLUSTERED INTERFACES FOR THE ALLEN-CAHN EQUATION
}

\author{
Andrea Malchiodi, Wei-Ming Ni And Juncheng Wei
}

\section{We consider the Allen-Cahn equation}

$$
\varepsilon^{2} \Delta u+u-u^{3}=0 \quad \text { in } \Omega, \quad \frac{\partial u}{\partial v}=0 \quad \text { on } \partial \Omega,
$$

where $\Omega=B_{1}(0)$ is the unit ball in $\mathbb{R}^{n}$ and $\varepsilon>0$ is a small parameter. We prove the existence of a radial solution $u_{\varepsilon}$ having $N$ interfaces $\left\{u_{\varepsilon}(r)=0\right\}=$ $\bigcup_{j=1}^{N}\left\{r=r_{j}^{\varepsilon}\right\}$, where $1>r_{1}^{\varepsilon}>r_{2}^{\varepsilon}>\cdots>r_{N}^{\varepsilon}$ are such that $1-r_{1}^{\varepsilon} \sim \varepsilon \log (1 / \varepsilon)$ and $r_{j-1}^{\varepsilon}-r_{j}^{\varepsilon} \sim \varepsilon \log (1 / \varepsilon)$ for $j=2, \ldots, N$. Moreover, the Morse index of $u_{\varepsilon}$ in $H_{r}^{1}\left(\Omega_{\varepsilon}\right)$ is exactly $N$.

\section{Introduction}

The aim of this paper is to construct a family of clustered transitional layered solutions to the Allen-Cahn equation

$$
\varepsilon^{2} \Delta u+u-u^{3}=0 \quad \text { in } \Omega \quad \text { and } \quad \frac{\partial u}{\partial v}=0 \quad \text { on } \partial \Omega,
$$

where $\Delta=\sum_{i=1}^{n} \partial^{2} / \partial x_{i}^{2}$ is the Laplace operator, $\Omega=B_{1}(0)$ is the unit ball in $\mathbb{R}^{n}$, $\varepsilon>0$ is a small parameter, and $\nu(x)$ denotes the unit outer normal at $x \in \partial \Omega$.

Problem (1-1) and its parabolic counterpart have been a subject of extensive research for many years. In order to describe some known results, we define the Allen-Cahn functional (see [Allen and Cahn 1979]),

$$
J_{\varepsilon}[u]=\int_{\Omega}\left(\frac{\varepsilon^{2}}{2}|\nabla u|^{2}-F(u)\right), \quad \text { where } F(u)=-\frac{1}{4}\left(1-u^{2}\right)^{2} .
$$

The set $\{x \in \Omega \mid u(x)=0\}$ is called the interface of $u$. Let $\operatorname{Per}_{\Omega}(A)$ be the relative perimeter of the set $A \subset \Omega$. Using $\Gamma$-convergence techniques (see [Modica 1987]), Kohn and Sternberg [1989] obtained a general result stating that in a neighborhood

MSC2000: primary 35B40, 35B45; secondary 35J40.

Keywords: boundary clustered interfaces, Allen-Cahn equation.

Malchiodi is supported by MURST, under the project Variational Methods and Nonlinear Differential Equations. He thanks the Department of Mathematics, Chinese University of Hong Kong, for its hospitality during his stay. Ni is partially supported by the NSF grant DMS 0400452. Wei's research is partially supported by an Earmarked Grant from RGC of Hong Kong. 
of an isolated local minimizer of $\operatorname{Per}_{\Omega}$ there exists a local minimizer to the functional $J_{\varepsilon}$. They further used this idea to show the existence of a stable solution for (1-1) in two-dimensional nonconvex domains, such as a dumb-bell. Since then, the existence of solutions with a single interface intersecting the boundary has been established and studied by many authors. See [Alikakos et al. 2000; Bronsard and Stoth 1996; Flores et al. 2001; Kowalczyk 2005; Padilla and Tonegawa 1998; Sternberg and Zumbrun 1998] and the references therein. However, the existence of multiple interfaces is only proved, in the one-dimensional case, for the AllenCahn equation (with inhomogeneous terms)

$$
\varepsilon^{2} u^{\prime \prime}+a(x)\left(u-u^{3}\right)=0, \quad-1<x<1, \quad u^{\prime}( \pm 1)=0
$$

(see [Nakashima 2003; Nakashima and Tanaka 2003]); and, in the higher-dimensional case, for the following nonlinear equation with bistable nonlinearity and inhomogeneous term:

$$
\varepsilon^{2} \Delta u+u(u-a(|x|))(1-u)=0 \quad \text { in } B_{1}(0), \quad \frac{\partial u}{\partial v}=0 \quad \text { on } \partial B_{1}(0)
$$

(see [Dancer and Yan 2003]). The result of this last paper states that if $a(r)$ has a critical point $r_{0} \in(0,1)$ such that $a\left(r_{0}\right)=1 / 2, a^{\prime}\left(r_{0}\right)=0, a^{\prime \prime}\left(r_{0}\right)<0$, then there exists a clustered interior-layer solution to (1-3). All three papers use the properties of the inhomogeneous terms to construct multiple (interior) interfaces. (For the Allen-Cahn equation with inhomogeneity, $\Delta u+a(x)\left(u-u^{3}\right)=0$ in $\mathbb{R}^{2}$, see [Rabinowitz and Stredulinsky 2003; 2004].)

Here, we continue our study, initiated in [Malchiodi et al. 2005], of clustered layered solutions for semilinear elliptic equations, and show that the homogeneous Allen-Cahn equation itself can generate multiple clustered interfaces near the boundary. In that paper we showed that the singularly-perturbed Neumann problem

$$
\left\{\begin{array}{l}
\varepsilon^{2} \Delta u-u+u^{p}=0 \text { in } \Omega, \\
u>0 \text { in } \Omega \text { and } \frac{\partial u}{\partial v}=0 \text { on } \partial \Omega,
\end{array}\right.
$$

has a clustered layered solution near the boundary. (The existence of a one-layer solution to (1-4) near the boundary was first established in [Ambrosetti et al. 2003; 2004].) The purpose of this paper is to show that a similar phenomenon happens to the Allen-Cahn equation. In particular, we establish the existence of clustered interfaces - the so-called "phantom interfaces" — in higher dimensions. Moreover, we show that, for each fixed positive integer $N$, there exists a solution to (1-1) with Morse index $N$ (in the space of radial functions).

Our main result is this: 
Theorem 1.1. Let $N$ be a fixed positive integer. There exists $\varepsilon_{N}>0$ such that, for all $\varepsilon<\varepsilon_{N}$, problem (1-1) admits a radially symmetric solution $u_{\varepsilon}$ with the following properties:

(1) the set of interfaces $\left\{u_{\varepsilon}(r)=0\right\}$ contains $N$ spheres $\left\{r=r_{j}^{\varepsilon}\right\}, j=1, \ldots, N$, with

$$
1-r_{1}^{\varepsilon} \sim \varepsilon \log \frac{1}{\varepsilon}, \quad r_{j-1}^{\varepsilon}-r_{j}^{\varepsilon} \sim \varepsilon \log \frac{1}{\varepsilon}, \quad j=2, \ldots, N .
$$

More precisely, we have $u_{\varepsilon}\left(r_{j}^{\varepsilon}+\varepsilon y\right) \rightarrow(-1)^{j} H(y)$, where $H(y)$ is the unique heteroclinic solution of

$$
H^{\prime \prime}+H-H^{3}=0, \quad H(0)=0, \quad H( \pm \infty)= \pm 1 .
$$

(2) The solution $u_{\varepsilon}$ has the energy bound

$$
J_{\varepsilon}\left[u_{\varepsilon}\right]=\omega_{n-1} N \varepsilon I[H]+o(\varepsilon),
$$

where

$$
I[H]=\int_{\mathbb{R}}\left(\frac{1}{2}\left(H^{\prime}\right)^{2}-F(H)\right),
$$

and where $\omega_{n-1}$ denotes the volume of $S^{n-1}$.

(3) The Morse index of $u_{\varepsilon}$ in $H_{r}^{1}(\Omega)$ is exactly $N$, where $H_{r}^{1}(\Omega)$ denotes the space of radial functions in $H^{1}(\Omega)$.

Remark 1.2. By a simple transformation, Theorem 1.1 readily extends to (1-3) with $a(r) \equiv 1 / 2$.

Our approach is similar to that of [Malchiodi et al. 2005], where a finite-dimensional reduction procedure combined with a variational approach is used. Such a method has been used successfully in many other papers, for example, [Ambrosetti et al. 2003; 2004; Dancer and Yan 1999; Gui and Wei 1999; 2000; Gui et al. 2000].

In the rest of section, we introduce some notation to be used later.

By the scaling $x=\varepsilon y$, problem (1-1) is reduced to the ODE

$$
\left\{\begin{array}{l}
u_{r r}+\frac{n-1}{r} u_{r}+f(u)=0 \quad \text { for } r \in(0,1 / \varepsilon), \\
u^{\prime}(0)=u^{\prime}(1 / \varepsilon)=0,
\end{array}\right.
$$

where $f(u)=u-u^{3}$. From now on, we will work with (1-8).

Let $H(y)$ be the unique solution to (1-6). Set

$$
\Omega_{\varepsilon}=(1 / \varepsilon) B_{1}(0)=B_{1 / \varepsilon}(0), \quad \text { and } \quad I_{\varepsilon}=(0,1 / \varepsilon) .
$$

For $u \in C^{2}\left(\Omega_{\varepsilon}\right)$ and $u=u(r)$, we have

$$
\Delta u=u^{\prime \prime}+\frac{n-1}{r} u^{\prime} .
$$


For $k \in \mathbb{N}$, we denote by $H_{r}^{k}\left(\Omega_{\varepsilon}\right)$ the space of radial functions in $H^{k}\left(\Omega_{\varepsilon}\right)$. On $H_{r}^{1}\left(\Omega_{\varepsilon}\right)$, we define an inner product as follows:

$$
(u, v)_{\varepsilon}=\int_{0}^{1 / \varepsilon}\left(u^{\prime} v^{\prime}+2 u v\right) r^{n-1} d r .
$$

Similarly, the inner product on $L_{r}^{2}\left(\Omega_{\varepsilon}\right)$ can be defined by

$$
\langle u, v\rangle_{\varepsilon}=\int_{0}^{1 / \varepsilon}(u v) r^{n-1} d r .
$$

We also introduce a new energy functional that, up to a positive multiplicative constant, is equivalent to $J_{\varepsilon}$ :

$$
\mathscr{E}_{\varepsilon}[u]=\frac{1}{2} \int_{0}^{1 / \varepsilon}\left|u^{\prime}\right|^{2} r^{n-1}-\int_{0}^{1 / \varepsilon} F(u) r^{n-1} d r, \quad u \in H_{r}^{1}\left(\Omega_{\varepsilon}\right) .
$$

Throughout this paper, unless otherwise stated, the letter $C$ will always denote various generic constants that are independent of $\varepsilon$, for $\varepsilon$ sufficiently small. The notation $A_{\varepsilon} \gg B_{\varepsilon}$ means that $\lim _{\varepsilon \rightarrow 0}\left|B_{\varepsilon}\right| /\left|A_{\varepsilon}\right|=0$, while $A_{\varepsilon} \ll B_{\varepsilon}$ means $\left(1 / A_{\varepsilon}\right) \gg$ $\left(1 / B_{\varepsilon}\right)$.

\section{Some preliminary analysis}

In this section we introduce a family of approximate solutions to (1-8) and derive some useful estimates.

Let $H$ be the unique solution of (1-6). It is easy to see that

$$
\begin{cases}H(y)-1=-A_{0} e^{-\sqrt{2}|y|}+O\left(e^{-2 \sqrt{2}|y|}\right) & \text { for } y>1, \\ H(y)+1=A_{0} e^{-\sqrt{2}|y|}+O\left(e^{-2 \sqrt{2}|y|}\right) & \text { for } y<-1, \\ H^{\prime}(y)=\sqrt{2} A_{0} e^{-\sqrt{2}|y|}+O\left(e^{-2 \sqrt{2}|y|}\right) & \text { for }|y|>1,\end{cases}
$$

where $A_{0}>0$ is a fixed constant.

We state the following well-known lemma on $H$. For a proof, see [Müller 1993, Lemma 4.1].

Lemma 2.1. For the eigenvalue problem

$$
\phi^{\prime \prime}+f^{\prime}(H) \phi=\lambda \phi, \quad|\phi| \leq 1, \quad \text { in } \mathbb{R},
$$

there holds

$$
\lambda_{1}=0, \quad \phi_{1}=c H^{\prime}, \quad \lambda_{2}<0 .
$$

For $u \in H_{r}^{2}\left(\Omega_{\varepsilon}\right)$, we define the operator

$$
\mathscr{S}_{\varepsilon}[u]:=u_{r r}+\frac{n-1}{r} u_{r}+f(u) .
$$


We introduce the set

$$
\Lambda=\left\{\begin{array}{l|l}
\mathbf{t}=\left(t_{1}, \ldots, t_{N}\right) & \begin{array}{l}
t_{N}>1-\varepsilon(\log (1 / \varepsilon))^{2}, \quad 1-t_{1} \geq \eta \varepsilon \log (1 / \varepsilon) \\
t_{j-1}-t_{j}>\eta \varepsilon \log (1 / \varepsilon), j=2, \ldots, N
\end{array}
\end{array}\right\},
$$

where $\eta \in(0,1 / 8 \sqrt{2})$ is a fixed number.

Let $\chi(s)$ be a cut-off function such that $\chi(s)=1$ for $s \leq 1 / 4$ and $\chi(s)=0$ for $s \geq 1 / 2$. For $t \in(3 / 4,1)$, we define

$$
\rho_{\varepsilon}(t)=H^{\prime}\left(\frac{1-t}{\varepsilon}\right) ; \quad \beta_{\varepsilon}(r)=\frac{1}{\sqrt{2}} e^{\sqrt{2}(r-1 / \varepsilon)}, \quad r \in[0,1 / \varepsilon],
$$

and

$$
\begin{aligned}
& H_{t}(r)=H\left(r-\frac{t}{\varepsilon}\right), \\
& H_{\varepsilon, t}(r)=\left(H\left(r-\frac{t}{\varepsilon}\right)-\rho_{\varepsilon}(t) \beta_{\varepsilon}(r)\right)(1-\chi(\varepsilon r))-\chi(\varepsilon r) .
\end{aligned}
$$

It is easy to see that, for $(1-t) / \varepsilon \gg 1$,

$$
\rho_{\varepsilon}(t)=\sqrt{2} A_{0} e^{-\sqrt{2}(1-t) / \varepsilon}+O\left(e^{-2 \sqrt{2}(1-t) / \varepsilon}\right) .
$$

We first assume that $N$ is odd. For $\mathbf{t} \in \Lambda$, we now define our approximate function:

If $N$ is even, we set

$$
H_{\varepsilon, \mathbf{t}}(r)=\sum_{j=1}^{N}(-1)^{j} H_{\varepsilon, t_{j}}(r)
$$

$$
H_{\varepsilon, \mathbf{t}}(r)=\sum_{j=1}^{N}(-1)^{j} H_{\varepsilon, t}(r)-1=\sum_{j=1}^{N+1}(-1)^{j} H_{\varepsilon, t_{j}}(r)
$$

where we use the convention that $H_{\varepsilon, t_{N+1}}=1$. So, without loss of generality, we can assume that $N$ is odd.

Note that, for $r \leq 1 /(2 \varepsilon)$, there holds

$$
\left|H_{\varepsilon, t}(r)-(-1)^{N}\right|+\left|H_{\varepsilon, t}^{\prime}(r)\right|+\left|H_{\varepsilon, t}^{\prime \prime}(r)\right| \leq e^{-1 /(C \varepsilon)} .
$$

Observe also that, by construction, $H_{\varepsilon, \mathbf{t}}$ satisfies the Neumann boundary condition, namely $H_{\varepsilon, \mathbf{t}}^{\prime}(0)=H_{\varepsilon, \mathbf{t}}^{\prime}(1 / \varepsilon)=0$. Furthermore, $H_{\varepsilon, \mathbf{t}}$ depends smoothly on $\mathbf{t}$ as a map with values in $C^{2}([0,1 / \varepsilon])$.

The next lemma shows that $H_{\varepsilon, \mathrm{t}}$ is a good approximate function to (1-8).

Lemma 2.2. For $\varepsilon$ sufficiently small and $\mathbf{t} \in \Lambda$, one has

$$
\begin{aligned}
\left\|\mathscr{S}_{\varepsilon}\left[H_{\varepsilon, \mathbf{t}}\right]\right\|_{L^{\infty}}+\varepsilon^{n-1} \int_{0}^{1 / \varepsilon} \mid \mathscr{S}_{\varepsilon}[ & \left.H_{\varepsilon, \mathbf{t}}\right] \mid r^{n-1} d r \\
& \leq C\left(\varepsilon+\sum_{j=1}^{N}\left(\rho_{\varepsilon}\left(t_{j}\right)\right)^{2}+\sum_{i \neq j} e^{-\sqrt{2}\left|t_{i}-t_{j}\right| / \varepsilon}\right) .
\end{aligned}
$$


Proof. Using (1-6) it is easy to see that

$$
\begin{aligned}
\mathscr{S}_{\varepsilon}\left[H_{\varepsilon, \mathbf{t}}\right]=\frac{n-1}{r} & H_{\varepsilon, \mathbf{t}}^{\prime}+f\left(H_{\varepsilon, \mathbf{t}}\right) \\
& \quad-\sum_{l=1}^{N}(-1)^{l} f\left(H_{t_{l}}\right)-2 \sum_{l=1}^{N}(-1)^{l} \rho_{\varepsilon}\left(t_{l}\right) \beta_{\varepsilon}(r)+O\left(e^{-1 /(C \varepsilon)}\right) .
\end{aligned}
$$

The first term in the right-hand side of (2-13) can be estimated as

$$
\frac{1}{r} H_{\varepsilon, \mathbf{t}}^{\prime}=\frac{1}{r} \sum_{j=1}^{N}(-1)^{j}\left(H_{t_{j}}^{\prime}-\rho_{\varepsilon}\left(t_{j}\right) \beta_{\varepsilon}^{\prime}(r)\right)+O\left(e^{-1 /(C \varepsilon)}\right) .
$$

From the decay of $H^{\prime}$ and $\beta_{\varepsilon}$ we deduce that

$$
\left\|\frac{1}{r} H_{\varepsilon, \mathbf{t}}^{\prime}\right\|_{\infty}+\varepsilon^{n-1} \int_{0}^{1 / \varepsilon} \frac{1}{r}\left|H_{\varepsilon, \mathbf{t}}^{\prime}\right| r^{n-1} d r \leq C \varepsilon .
$$

Next, we note that

$$
\left|f\left(H_{\varepsilon, \mathbf{t}}\right)-\sum_{l=1}^{N} f\left((-1)^{l} H_{t_{l}}\right)-2 \sum_{l=1}^{N}(-1)^{l} \rho_{\varepsilon}\left(t_{l}\right) \beta_{\varepsilon}(r)\right| \leq S_{1}+S_{2},
$$

where

$$
\begin{aligned}
& S_{1}=\left|f\left(\sum_{j=1}^{N}(-1)^{j} H_{t_{j}}\right)-\sum_{j=1}^{N} f\left((-1)^{j} H_{t_{j}}\right)\right|, \\
& S_{2}=\left|f\left(\sum_{j=1}^{N}(-1)^{j} H_{\varepsilon, t_{j}}\right)-f\left(\sum_{j=1}^{N}(-1)^{j} H_{t_{j}}\right)-2 \sum_{j=1}^{N}(-1)^{j} \rho_{\varepsilon}\left(t_{j}\right) \beta_{\varepsilon}(r)\right| .
\end{aligned}
$$

To estimate $S_{1}$ and $S_{2}$, we divide the domain $I_{\varepsilon}=(0,1 / \varepsilon)$ into the $N$ intervals $I_{\varepsilon, 1}, \ldots, I_{\varepsilon, N}$ defined by

$$
\begin{aligned}
& I_{\varepsilon, 1}=\left[\frac{t_{1}+t_{2}}{2 \varepsilon}, \frac{1}{\varepsilon}\right), \quad I_{\varepsilon, j}=\left[\frac{t_{j}+t_{j+1}}{2 \varepsilon}, \frac{t_{j}+t_{j-1}}{2 \varepsilon}\right), j=2, \ldots, N-1, \\
& \text { and } I_{\varepsilon, N}=\left(0, \frac{t_{N}+t_{N-1}}{2 \varepsilon}\right) .
\end{aligned}
$$

We choose $t_{0}=2-t_{1}$ and $t_{N+1}=-t_{N}$, so that

$$
I_{\varepsilon, j}=\left[\frac{t_{j}+t_{j+1}}{2 \varepsilon}, \frac{t_{j}+t_{j-1}}{2 \varepsilon}\right), \quad j=1, \ldots, N, \quad I_{\varepsilon}=\bigcup_{j=1}^{N} I_{\varepsilon, j} .
$$

For $r \in I_{\varepsilon, j}$ and $j<l$, we note that

while for $j>l$,

$$
H_{t_{l}}(r)=1+O\left(e^{-\sqrt{2}\left|r-t_{j} / \varepsilon\right|}\right)
$$

$$
H_{t_{l}}(r)=-1+O\left(e^{-\sqrt{2}\left|r-t_{j} / \varepsilon\right|}\right)
$$


Since $N$ is odd, we see that

$$
\sum_{l \neq j}(-1)^{l} H_{t_{l}}=\sum_{l<j}(-1)^{l}\left(H_{t_{l}}+1\right)+\sum_{l>j}(-1)^{l}\left(H_{t_{l}}-1\right) .
$$

Thus, we can rewrite $S_{1}$ as:

$$
\begin{array}{r}
S_{1}=f\left(\sum_{l<j}(-1)^{l}\left(H_{t_{l}}+1\right)+(-1)^{j} H_{t_{j}}+\sum_{l>j}(-1)^{l}\left(H_{t_{l}}-1\right)\right)-(-1)^{j} f\left(H_{t_{j}}\right) \\
-\sum_{l \neq j}(-1)^{l} f\left(H_{t_{l}}\right) \\
=f^{\prime}\left((-1)^{j} H_{t_{j}}\right)\left(\sum_{l<j}(-1)^{l}\left(H_{t_{l}}+1\right)+\sum_{l>j}(-1)^{l}\left(H_{t_{l}}-1\right)\right)-\sum_{l \neq j}(-1)^{l} f\left(H_{t_{l}}\right) \\
+O\left(\sum_{l<j}\left(H_{t_{l}}+1\right)^{2}+\sum_{l>j}\left(H_{t_{l}}-1\right)^{2}\right)
\end{array}
$$

This quantity can also be written

$$
\begin{aligned}
S_{1}=\left(f^{\prime}\left((-1)^{j} H_{t_{j}}\right)-f^{\prime}(1)\right)\left(\sum _ { l < j } ( - 1 ) ^ { l } \left(H_{t_{l}}\right.\right. & \left.+1)+\sum_{l>j}(-1)^{l}\left(H_{t_{l}}-1\right)\right) \\
& +O\left(\sum_{l<j}\left(H_{t_{l}}+1\right)^{2}+\sum_{l>j}\left(H_{t_{l}}-1\right)^{2}\right) \\
=O\left(\min \left\{H_{t_{j}}+1, H_{t_{j}}-1\right\}\right)\left(\sum_{l<j}\left(H_{t_{l}}+1\right)\right. & \left.+\sum_{l>j}\left(H_{t_{l}}-1\right)\right) \\
& +O\left(\sum_{l<j}\left(H_{t_{l}}+1\right)^{2}+\sum_{l>j}\left(H_{t_{l}}-1\right)^{2}\right) .
\end{aligned}
$$

Then, with some elementary computations, one finds that

$$
\left\|S_{1}\right\|_{L^{\infty}\left(I_{\varepsilon, j}\right)}+\varepsilon^{n-1} \int_{I_{\varepsilon, j}}\left|S_{1}(r)\right| r^{n-1} d r \leq C \sum_{i \neq j} e^{-\sqrt{2}\left|t_{i}-t_{j}\right| / \varepsilon} .
$$

It remains to estimate $S_{2}$. For this, we note that, for $r \in I_{\varepsilon, j}$ and $j \geq 2$, we have

$$
\rho_{\varepsilon}\left(t_{j}\right) \beta_{\varepsilon}(r)=O\left(e^{-\sqrt{2}\left(1-t_{1}\right) / \varepsilon} e^{\sqrt{2}(r-1 / \varepsilon)}\right),
$$

from which it follows that, for $j \geq 2$,

$$
\left\|S_{2}\right\|_{L^{\infty}\left(I_{\varepsilon, j}\right)}+\varepsilon^{n-1} \int_{I_{\varepsilon, j}}\left|S_{2}(r)\right| r^{n-1} d r=O\left(e^{-2 \sqrt{2}\left(1-t_{1}\right) / \varepsilon}\right)=O\left(\sum_{j=1}^{N}\left(\rho_{\varepsilon}\left(t_{j}\right)\right)^{2}\right) .
$$

Therefore, we just need to consider the case when $r \in I_{\varepsilon, 1}$. But, since $f^{\prime}( \pm 1)=-2$, we have

$$
\begin{aligned}
S_{2}=f\left(\sum_{l=1}^{N}(-1)^{l} H_{t_{l}}-\sum_{l=1}^{N}(-1)^{l}\right. & \left.\rho_{\varepsilon}\left(t_{l}\right) \beta_{\varepsilon}(r)\right) \\
& -f\left(\sum_{l=1}^{N}(-1)^{l} H_{t_{l}}\right)-f^{\prime}(-1) \sum_{l=1}^{N}(-1)^{l} \rho_{\varepsilon}\left(t_{l}\right) \beta_{\varepsilon}(r)
\end{aligned}
$$




$$
\begin{aligned}
& =\left(f^{\prime}\left(\sum_{l=1}^{N}(-1)^{l} H_{t_{l}}\right)-f^{\prime}(-1)\right) \sum_{l=1}^{N}(-1)^{l} \rho_{\varepsilon}\left(t_{l}\right) \beta_{\varepsilon}(r)+O\left(\sum_{l=1}^{N} \rho_{\varepsilon}\left(t_{l}\right)^{2} \beta_{\varepsilon}(r)^{2}\right) \\
& =O\left(\sum_{l=1}^{N} e^{-\sqrt{2}\left|r-t_{l} / \varepsilon\right|}\right)\left(\sum_{l=1}^{N} \rho_{\varepsilon}\left(t_{l}\right) \beta_{\varepsilon}(r)\right)+O\left(\sum_{l=1}^{N} \rho_{\varepsilon}\left(t_{l}\right)^{2} \beta_{\varepsilon}(r)^{2}\right) .
\end{aligned}
$$

Hence, we also get

$$
\left\|S_{2}\right\|_{L^{\infty}\left(I_{1}\right)}+\varepsilon^{n-1} \int_{I_{1}}\left|S_{2}(r)\right| r^{n-1} d r \leq C \rho_{\varepsilon}^{2}\left(t_{1}\right) .
$$

The proof of the next lemma is postponed to the appendix.

Lemma 2.3. Let $\mathbf{t} \in \Lambda$. For $\varepsilon$ sufficiently small, we have

$$
\begin{aligned}
\mathscr{E}_{\varepsilon}\left[\sum_{j=1}^{N}(-1)^{j} H_{\varepsilon, t_{j}}\right] & \\
=I[H] & \sum_{i=1}^{N}\left(\frac{t_{i}}{\varepsilon}\right)^{n-1}-\left(\frac{1}{\varepsilon}\right)^{n-1}\left(\sqrt{2} A_{0}^{2}+o(1)\right) e^{-2 \sqrt{2}\left(1-t_{1}\right) / \varepsilon} \\
& -\sum_{j=2}^{N}\left(\frac{t_{j}}{\varepsilon}\right)^{n-1}\left(\sqrt{2} A_{0}^{2}+o(1)\right) e^{-\sqrt{2}\left|t_{j}-t_{j-1}\right| / \varepsilon}+O\left(\varepsilon^{2-n}\right),
\end{aligned}
$$

where $A_{0}>0$ is defined in (2-1).

\section{Lyapunov-Schmidt process: finite-dimensional reduction}

In this section we outline the so-called Lyapunov-Schmidt reduction process. Since this can be proved along the same ideas of [Malchiodi et al. 2005, Sections 3], we skip some of the details.

Fix $\mathbf{t} \in \Lambda$. Integrating by parts, one can show that orthogonality to $\partial H_{\varepsilon, t_{j}} / \partial t_{j}$ in $H_{r}^{1}\left(\Omega_{\varepsilon}\right), j=1, \ldots, N$, is equivalent to orthogonality in $L^{2}\left(\Omega_{\varepsilon}\right)$ to the functions

$$
Z_{\varepsilon, t_{j}}=\Delta\left(\frac{\partial H_{\varepsilon, t_{j}}}{\partial t_{j}}\right)-2 \frac{\partial H_{\varepsilon, t_{j}}}{\partial t_{j}}, \quad j=1, \ldots, N
$$

By elementary computations, differentiating (1-6) we obtain

$$
\begin{aligned}
\frac{\partial H_{\varepsilon, t_{j}}}{\partial t_{j}} & =-\frac{1}{\varepsilon} H^{\prime}\left(r-\frac{t_{j}}{\varepsilon}\right)+\frac{1}{\varepsilon} H^{\prime \prime}\left(\frac{1-t_{j}}{\varepsilon}\right) \beta_{\varepsilon}(r)+O\left(e^{-1 /(C \varepsilon)}\right), \\
Z_{\varepsilon, t_{j}} & =\left(f^{\prime}\left(H_{t_{j}}\right)-f^{\prime}( \pm 1)\right) \frac{\partial H_{\varepsilon, t_{j}}}{\partial t_{j}}+\frac{n-1}{r}\left(\frac{\partial H_{\varepsilon, t_{j}}}{\partial t_{j}}\right)^{\prime} \\
& =-\frac{1}{\varepsilon} H_{t_{j}}^{\prime}\left(f^{\prime}\left(H_{t_{j}}\right)-f^{\prime}( \pm 1)\right)+o(1 / \varepsilon),
\end{aligned}
$$

where $O\left(e^{-1 /(C \varepsilon)}\right)$ and $o(1 / \varepsilon)$ are intended both in the $C^{1}$ and $H_{r}^{1}$ sense. 
We consider first the following linear problem: Given $h \in L^{\infty}\left(\Omega_{\varepsilon}\right)$, find a function $\phi$ satisfying

$$
\left\{\begin{array}{l}
L_{\varepsilon}[\phi]:=\phi^{\prime \prime}+\frac{n-1}{r} \phi^{\prime}+f^{\prime}\left(H_{\varepsilon, \mathbf{t}}\right) \phi=h+\sum_{j=1}^{N} c_{j} Z_{\varepsilon, t_{j}} \\
\phi^{\prime}(0)=\phi^{\prime}(1 / \varepsilon)=0 \quad \text { and } \quad\left\langle\phi, Z_{\varepsilon, t_{j}}\right\rangle_{\varepsilon}=0, \quad j=1, \ldots, N,
\end{array}\right.
$$

for some constants $c_{j}, j=1, \ldots, N$. For this, define the norm

$$
\|\phi\|_{*}=\sup _{r \in(0,1 / \varepsilon)}|\phi(r)| .
$$

Assuming a solution to (3-4) exists, we have an estimate on $\phi$ :

Proposition 3.1. Let $\phi$ satisfy (3-4). For $\varepsilon$ sufficiently small, we have

$$
\|\phi\|_{*} \leq C\|h\|_{*},
$$

where $C$ is a positive constant independent of $\varepsilon$ and $\mathbf{t} \in \Lambda$.

Proof. The argument is similar in spirit of that of [Malchiodi et al. 2005, Proposition 3.1]. For the sake of completeness, we include a proof here.

Arguing by contradiction, assume that

$$
\|\phi\|_{*}=1, \quad\|h\|_{*}=o(1) .
$$

We multiply (3-4) by $\partial H_{\varepsilon, t_{j}} / \partial t_{j}$ and integrate over $\Omega_{\varepsilon}$ to obtain

$$
\sum_{i=1}^{N} c_{i}\left\langle Z_{\varepsilon, t_{i}}, \frac{\partial H_{\varepsilon, t_{j}}}{\partial t_{j}}\right\rangle_{\varepsilon}=-\left\langle h, \frac{\partial H_{\varepsilon, t_{j}}}{\partial t_{j}}\right\rangle_{\varepsilon}+\left\langle\Delta \phi+f^{\prime}\left(H_{\varepsilon, \mathbf{t}}\right) \phi, \frac{\partial H_{\varepsilon, t_{j}}}{\partial t_{j}}\right\rangle_{\varepsilon} .
$$

From the exponential decay of $H^{\prime}$, one finds

$$
\left\langle h, \frac{\partial H_{\varepsilon, t_{j}}}{\partial t_{j}}\right\rangle_{\varepsilon}=\int_{0}^{1 / \varepsilon} h \frac{\partial H_{\varepsilon, t_{j}}}{\partial t_{j}} r^{n-1} d r=O\left(\|h\|_{*} \varepsilon^{-n}\right) .
$$

Moreover, integrating by parts and using (3-2) and (3-3), we deduce

$$
\left\langle\Delta \phi+f^{\prime}\left(H_{\varepsilon, \mathbf{t}}\right) \phi, \frac{\partial H_{\varepsilon, t_{j}}}{\partial t_{j}}\right\rangle_{\varepsilon}=\left\langle Z_{\varepsilon, t_{j}}+f^{\prime}\left(H_{\varepsilon, \mathbf{t}}\right) \frac{\partial H_{\varepsilon, t_{j}}}{\partial t_{j}}, \phi\right\rangle_{\varepsilon}=o\left(\varepsilon^{-n}\|\phi\|_{*}\right) .
$$

From (3-2) and (3-3), we also see that

$$
\left\langle Z_{\varepsilon, t_{i}}, \frac{\partial H_{\varepsilon, t_{j}}}{\partial t_{j}}\right\rangle_{\varepsilon}=-\varepsilon^{-n-1}\left(t_{i}^{n-1} \delta_{i j} \int_{\mathbb{R}} f^{\prime}(H)\left(H^{\prime}\right)^{2}+o(1)\right),
$$

where $\delta_{i j}$ denotes the Kronecker symbol. Note that, using the equation $H^{\prime \prime \prime}+$ $f^{\prime}(H) H^{\prime}=0$, we find

$$
\int_{\mathbb{R}} f^{\prime}(H)\left(H^{\prime}\right)^{2}=\int_{\mathbb{R}}\left(H^{\prime \prime}\right)^{2}>0 .
$$


This shows that the left-hand side of the equation (3-8) is diagonally dominant in the indices $i$ and $j$, and hence, by (3-7), we have

$$
c_{i}=O\left(\varepsilon\|h\|_{*}\right)+o\left(\varepsilon\|\phi\|_{*}\right)=o(\varepsilon), \quad i=1, \ldots, N .
$$

Also, since we are assuming that $\|h\|_{*}=o(1)$ and since $\left\|Z_{\varepsilon, t_{j}}\right\|_{*}=O(1 / \varepsilon)$, there holds

$$
\left\|h+\sum_{j=1}^{N} c_{j} Z_{\varepsilon, t_{j}}\right\|_{*}=o(1)
$$

Thus, (3-4) yields

$$
\left\{\begin{array}{l}
\phi^{\prime \prime}+\frac{n-1}{r} \phi^{\prime}+f^{\prime}( \pm 1)+\left(f^{\prime}\left(H_{\varepsilon, \mathbf{t}}\right)-f^{\prime}( \pm 1)\right) \phi=o(1), \\
\phi^{\prime}(0)=\phi^{\prime}(1 / \varepsilon)=0 \quad \text { and } \quad\left\langle\phi, Z_{\varepsilon, t_{j}}\right\rangle_{\varepsilon}=0, \quad j=1, \ldots, N,
\end{array}\right.
$$

where $o(1)$ is in the sense of $L^{\infty}(0,1 / \varepsilon)$.

We show that (3-12) is incompatible with our assumption that $\|\phi\|_{*}=1$. First, we claim that

$$
|\phi| \rightarrow 0 \quad \text { on } \quad y \in \bigcup_{j=1}^{N}\left(\frac{t_{j}}{\varepsilon}-R, \frac{t_{j}}{\varepsilon}+R\right), \quad \text { as } \varepsilon \rightarrow 0,
$$

where $R$ is any fixed positive constant.

Indeed, assuming the contrary, there exist $\delta_{0}>0, j \in\{1, \ldots, N\}$, and sequences $\varepsilon_{k}, \phi_{k}, y_{k} \in\left(t_{j} / \varepsilon-R, t_{j} / \varepsilon+R\right)$ such that $\phi_{k}$ satisfies (3-4) and

$$
\left|\phi_{k}\left(y_{k}\right)\right| \geq \delta_{0} .
$$

Let $\tilde{\phi}_{k}=\phi_{k}\left(y-t_{j} / \varepsilon_{k}\right)$. Then, using (3-12) and $\|\phi\|_{*}=1$, as $\varepsilon_{k} \rightarrow 0, \tilde{\phi}_{k}$ converges weakly in $H_{\text {loc }}^{2}(\mathbb{R})$ and strongly in $C_{\text {loc }}^{1}(\mathbb{R})$ to a bounded function $\phi_{0}$ which satisfies

$$
\phi_{0}^{\prime \prime}+f^{\prime}(H) \phi_{0}=0 \quad \text { in } \mathbb{R}, \quad\left|\phi_{0}\right| \leq C .
$$

By Lemma 2.1, we have $\phi_{0}=c H^{\prime}$ for some $c$. Since $\tilde{\phi}_{k} \perp Z_{\varepsilon, t_{j}}$, we conclude that

$$
\int_{\mathbb{R}} \phi_{0} f^{\prime}(H)\left(H^{\prime}\right)^{2}(y)=0,
$$

which yields $c=0$. Hence $\phi_{0}=0$ and $\tilde{\phi}_{k} \rightarrow 0$ in $B_{2 R}(0)$. This contradicts (3-14), so (3-13) holds true.

Given $\delta>0$, the decay of $f^{\prime}(H)-f^{\prime}( \pm 1)$ together with (3-13) (with $R$ sufficiently large) imply that

$$
\left\|\left(f^{\prime}\left(H_{\varepsilon, \mathbf{t}}\right)-f^{\prime}( \pm 1)\right) \phi\right\|_{*} \leq \delta+\frac{1}{2}\|\phi\|_{*} .
$$


Using (3-12) and the Maximum Principle, one finds

$$
\|\phi\|_{*} \leq\left\|\left(f^{\prime}\left(H_{\varepsilon, \mathbf{t}}\right)-f^{\prime}( \pm 1)\right) \phi\right\|_{*}+\sum_{j=1}^{N}\left|c_{j}\right|\left\|Z_{\varepsilon, t_{j}}\right\|_{*}+\|h\|_{*} \leq 2 \delta+\frac{1}{2}\|\phi\|_{*},
$$

and hence

$$
\|\phi\|_{*} \leq 4 \delta<1,
$$

if we choose $\delta<1 / 4$. This contradicts (3-7).

Next, we consider the following nonlinear problem: Find a function $\phi$ such that for some constants $c_{j}, j=1, \ldots, N$, the equation

$$
\left\{\begin{array}{l}
\Delta\left(H_{\varepsilon, \mathbf{t}}+\phi\right)+f\left(H_{\varepsilon, \mathbf{t}}+\phi\right)=\sum_{j=1}^{N} c_{j} Z_{\varepsilon, t_{j}} \text { in } \Omega_{\varepsilon}, \\
\phi^{\prime}(0)=\phi^{\prime}\left(\frac{1}{\varepsilon}\right)=0 \quad \text { and } \quad\left\langle\phi, Z_{\varepsilon, t_{j}}\right\rangle_{\varepsilon}=0, \quad j=1, \ldots, N .
\end{array}\right.
$$

holds true.

The proof of the next result follows the same lines of [Malchiodi et al. 2005, Proposition 4.2].

Proposition 3.2. For $\mathbf{t} \in \Lambda$ and $\varepsilon$ sufficiently small, there exists a unique $\phi=\phi_{\varepsilon, \mathbf{t}}$ such that (3-16) holds. Moreover, $t \mapsto \phi_{\varepsilon, \mathrm{t}}$ is of class $C^{1}$ as a map into $H_{r}^{1}\left(\Omega_{\varepsilon}\right)$, and we have

$$
\left\|\phi_{\varepsilon, \mathbf{t}}\right\|_{*} \leq C\left(\varepsilon+\sum_{j=1}^{n} e^{-(3 / 2) \sqrt{2}\left(1-t_{j}\right) / \varepsilon}+\sum_{i \neq j} e^{-(3 / 4) \sqrt{2}\left|t_{i}-t_{j}\right| / \varepsilon}\right) .
$$

\section{Energy computation for reduced energy functional}

We expand the quantity

$$
\mathcal{M}_{\varepsilon}(\mathbf{t}):=\varepsilon^{n-1 \mathscr{E}_{\varepsilon}}\left[H_{\varepsilon, \mathbf{t}}+\phi_{\varepsilon, \mathbf{t}}\right]: \Lambda \rightarrow \mathbb{R}
$$

in $\varepsilon$ and $\mathbf{t}$, where $\phi_{\varepsilon, \mathbf{t}}$ is given by Proposition 3.2. Up to negligible error terms, the same expansion of Lemma 2.3 holds true.

Lemma 4.1. For $\mathbf{t} \in \Lambda$ and $\varepsilon$ sufficiently small, we have

$$
\begin{aligned}
\mathcal{M}_{\varepsilon}(\mathbf{t})=\varepsilon^{n-1} \mathscr{\mathscr { E }}_{\varepsilon}\left[H_{\varepsilon, \mathbf{t}}+\phi_{\varepsilon, \mathbf{t}}\right] & \\
=I[H] \sum_{j=1}^{N} t_{j}^{n-1}- & \left(\sqrt{2} A_{0}^{2}+o(1)\right) e^{-2 \sqrt{2}\left(1-t_{1}\right) / \varepsilon} \\
& -\left(\sqrt{2} A_{0}^{2}+o(1)\right) \sum_{j=2}^{N} t_{j}^{n-1} e^{-\sqrt{2}\left|t_{j}-t_{j-1}\right| / \varepsilon}+O(\varepsilon) .
\end{aligned}
$$


Proof. It is sufficient to show that

$$
\mu_{\varepsilon}(\mathbf{t})=\varepsilon^{n-1 \mathscr{E}_{\varepsilon}}\left[H_{\varepsilon, \mathbf{t}}\right]+o\left(\sum_{j=1}^{N} e^{-2 \sqrt{2}\left(1-t_{j}\right) / \varepsilon}+\sum_{i \neq j} e^{-\sqrt{2}\left|t_{i}-t_{j}\right| / \varepsilon}\right)+O(\varepsilon),
$$

and to apply Lemma 2.3. In order to do this, we write

$$
\varepsilon^{1-n} \mathcal{M}_{\varepsilon}=\mathscr{E}_{\varepsilon}\left[H_{\varepsilon, \mathbf{t}}\right]+K_{1}+K_{2}-K_{3},
$$

where

$$
\begin{aligned}
& K_{1}=\int_{0}^{1 / \varepsilon}\left(H_{\varepsilon, \mathbf{t}}^{\prime} \phi_{\varepsilon, \mathbf{t}}^{\prime}-f\left(H_{\varepsilon, \mathbf{t}}\right) \phi_{\varepsilon, \mathbf{t}}\right) r^{n-1} d r \\
& K_{2}=\frac{1}{2} \int_{0}^{1 / \varepsilon}\left(\left|\phi_{\varepsilon, \mathbf{t}}^{\prime}\right|^{2}-f^{\prime}\left(H_{\varepsilon, \mathbf{t}}\right) \phi_{\varepsilon, \mathbf{t}}^{2}\right) r^{n-1} d r ; \\
& K_{3}=\int_{0}^{1 / \varepsilon}\left(F\left(H_{\varepsilon, \mathbf{t}}+\phi_{\varepsilon, \mathbf{t}}\right)-F\left(H_{\varepsilon, \mathbf{t}}\right)-f\left(H_{\varepsilon, \mathbf{t}}\right) \phi_{\varepsilon, \mathbf{t}}-\frac{1}{2} f^{\prime}\left(H_{\varepsilon, \mathbf{t}}\right) \phi_{\varepsilon, \mathbf{t}}^{2}\right) r^{n-1} d r .
\end{aligned}
$$

Integrating by parts, using Lemma 2.2 and Proposition 3.1, we find

$$
\begin{array}{r}
\left|K_{1}\right|=\left|\int_{0}^{1 / \varepsilon} \mathscr{S}_{\varepsilon}\left[H_{\varepsilon, \mathbf{t}}\right] \phi_{\varepsilon, \mathbf{t}} r^{n-1} d r\right| \leq C\left\|\phi_{\varepsilon, \mathbf{t}}\right\|_{*} \int_{0}^{1 / \varepsilon}\left|\mathscr{S}_{\varepsilon}\left[H_{\varepsilon, \mathbf{t}}\right]\right| r^{n-1} d r \\
\leq C \varepsilon^{1-n}\left(\varepsilon^{2}+\sum_{j=1}^{N}\left(\rho_{\varepsilon}\left(t_{j}\right)\right)^{2+3 / 2}+\sum_{i \neq j} e^{-(7 / 4) \sqrt{2}\left|t_{i}-t_{j}\right| / \varepsilon}\right) .
\end{array}
$$

To estimate $K_{2}$, we note that $\phi_{\varepsilon, \mathbf{t}}$ satisfies

$$
\Delta \phi_{\varepsilon, \mathbf{t}}+f\left(H_{\varepsilon, \mathbf{t}}+\phi_{\varepsilon, \mathbf{t}}\right)-f\left(H_{\varepsilon, \mathbf{t}}\right)+\mathscr{Y}_{\varepsilon}\left[w_{\varepsilon, \mathbf{t}}\right]=\sum_{j=1}^{N} c_{j} Z_{\varepsilon, t_{j}} .
$$

Multiplying (4-4) by $\phi_{\varepsilon, t} r^{n-1}$ and integrating over $I_{\varepsilon}$, we obtain

$$
\begin{aligned}
\int_{I_{\varepsilon}} \mathscr{Y}_{\varepsilon}\left[H_{\varepsilon, \mathbf{t}}\right] \phi_{\varepsilon, \mathbf{t}} & r^{n-1} d r=\int_{I_{\varepsilon}}\left(\left|\phi_{\varepsilon, \mathbf{t}}^{\prime}\right|^{2}-f^{\prime}\left(H_{\varepsilon, \mathbf{t}}\right) \phi_{\varepsilon, \mathbf{t}}^{2}\right) r^{n-1} d r \\
& +\int_{I_{\varepsilon}}\left(f\left(H_{\varepsilon, \mathbf{t}}+\phi_{\varepsilon, \mathbf{t}}\right)-f\left(H_{\varepsilon, \mathbf{t}}\right)-f^{\prime}\left(H_{\varepsilon, \mathbf{t}}\right) \phi_{\varepsilon, \mathbf{t}}\right) \phi_{\varepsilon, \mathbf{t}} r^{n-1} d r .
\end{aligned}
$$

Hence, we find

$$
\begin{aligned}
2 K_{2}=-\int_{I_{\varepsilon}}\left(f\left(H_{\varepsilon, \mathbf{t}}+\phi_{\varepsilon, \mathbf{t}}\right)-f\left(H_{\varepsilon, \mathbf{t}}\right)-f^{\prime}\left(H_{\varepsilon, \mathbf{t}}\right) \phi_{\varepsilon, \mathbf{t}}\right) \phi_{\varepsilon, \mathbf{t}} r^{n-1} d r & \\
& +\int_{I_{\varepsilon}} \mathscr{S}_{\varepsilon}\left[H_{\varepsilon, \mathbf{t}}\right] \phi_{\varepsilon, \mathbf{t}} r^{n-1} d r .
\end{aligned}
$$

From Taylor's formula, we get

$$
\left|f\left(H_{\varepsilon, \mathbf{t}}+\phi_{\varepsilon, \mathbf{t}}\right)-f\left(H_{\varepsilon, \mathbf{t}}\right)-f^{\prime}\left(H_{\varepsilon, \mathbf{t}}\right) \phi_{\varepsilon, \mathbf{t}}\right| \leq C\left|\phi_{\varepsilon, \mathbf{t}}\right|^{2},
$$


so we deduce

$$
\left|K_{2}\right| \leq C \int_{I_{\varepsilon}}\left|\phi_{\varepsilon, \mathbf{t}}\right|^{3} r^{n-1} d r+C\left\|\phi_{\varepsilon, \mathbf{t}}\right\|_{*} \int_{I_{\varepsilon}} \mathscr{S}_{\varepsilon}\left[H_{\varepsilon, \mathbf{t}}\right] r^{n-1} d r .
$$

From the exponential decay of $H( \pm y)-( \pm 1)$ one finds that $\phi_{\varepsilon, \mathbf{t}}(r)$ satisfies

$$
\begin{aligned}
& \phi_{\varepsilon, \mathbf{t}}^{\prime \prime}+\frac{n-1}{r} \phi_{\varepsilon, \mathbf{t}}^{\prime}+f\left(H_{\varepsilon, \mathbf{t}}+\phi_{\varepsilon, \mathbf{t}}\right)-f\left(H_{\varepsilon, \mathbf{t}}\right)=O\left(\sum_{j=1}^{N} e^{-\sqrt{2}\left|r-t_{j} / \varepsilon\right|}\right), \\
& \phi_{\varepsilon, \mathbf{t}}^{\prime}(0)=\phi_{\varepsilon, \mathbf{t}}^{\prime}(1 / \varepsilon)=0 .
\end{aligned}
$$

From (4-4) and a comparison principle, we obtain

$$
\left|\phi_{\varepsilon, \mathbf{t}}(r)\right| \leq C \sum_{j=1}^{N} e^{-(\sqrt{2} / \tilde{C})\left|r-t_{j} / \varepsilon\right|}
$$

for some $\tilde{C}<1$.

Using Proposition 3.2 and (4-6), we get

$$
\left|K_{2}\right| \leq C \varepsilon^{1-n}\left(\varepsilon^{2}+\sum_{j=1}^{N}\left(\rho_{\varepsilon}\left(t_{j}\right)\right)^{3}+\sum_{i \neq j} e^{-2 \sqrt{2}\left|t_{i}-t_{j}\right| / \varepsilon}\right) .
$$

From the Hölder continuity of $f^{\prime}$, we deduce

$$
\left|F\left(H_{\varepsilon, \mathbf{t}}+\phi_{\varepsilon, \mathbf{t}}\right)-F\left(H_{\varepsilon, \mathbf{t}}\right)-f\left(H_{\varepsilon, \mathbf{t}}\right) \phi_{\varepsilon, \mathbf{t}}-\frac{1}{2} f^{\prime}\left(H_{\varepsilon, \mathbf{t}}\right) \phi_{\varepsilon, \mathbf{t}}^{2}\right| \leq C\left|\phi_{\varepsilon, \mathbf{t}}\right|^{3},
$$

so, again, it follows that

$$
\left|K_{3}\right| \leq C \varepsilon^{1-n}\left(\varepsilon^{2}+\sum_{j=1}^{N}\left(\rho_{\varepsilon}\left(t_{j}\right)\right)^{3}+\sum_{i \neq j} e^{-2 \sqrt{2}\left|t_{i}-t_{j}\right| / \varepsilon}\right) .
$$

Combining with (2-20) of Lemma 2.2, we obtain the conclusion.

\section{Proof of Theorem 1.1}

In this section we prove Theorem 1.1. Fix $\mathbf{t} \in \bar{\Lambda}$ and let $\phi_{\varepsilon, \mathbf{t}}$ be given by Proposition 3.2. Let also $M_{\varepsilon}(\mathbf{t})$ denote the reduced energy functional defined by (4-1).

Proposition 5.1. For $\varepsilon$ small, the following maximization problem

$$
\sup \left\{M_{\varepsilon}(\mathbf{t}) \mid \mathbf{t} \in \Lambda\right\}
$$

has a solution $\mathbf{t}^{\varepsilon}$ in the interior of $\Lambda$.

Proof. Since $\mu_{\varepsilon}(\mathbf{t})$ is continuous in $\mathbf{t}$, it achieves a maximum in $\bar{\Lambda}$. Let $\mathbf{t}^{\varepsilon}$ be a maximum point. We claim that $\mathbf{t}^{\varepsilon} \in \Lambda$.

We argue by contradiction and assume that $\mathfrak{t}^{\varepsilon} \in \partial \Lambda$. From the definition of $\Lambda$, there are three possibilities: either $1-t_{1}=\eta \varepsilon \log (1 / \varepsilon)$, or there exists $j \geq 2$ such that $t_{j-1}-t_{j}=\eta \varepsilon \log (1 / \varepsilon)$, or, finally, $t_{N}=1-\varepsilon(\log (1 / \varepsilon))^{2}$. 
In the first case, we have

$$
\begin{aligned}
I[H] t_{1}^{n-1}-\left(\sqrt{2} A_{0}^{2}+\right. & o(1)) e^{-2 \sqrt{2}\left(1-t_{1}\right) / \varepsilon} \\
& =I[H]\left(1-\eta \varepsilon \log \frac{1}{\varepsilon}\right)^{n-1}-\sqrt{2} A_{0}^{2} e^{-2 \eta \sqrt{2} \log (1 / \varepsilon)}+o\left(\varepsilon^{2 \sqrt{2} \eta}\right) \\
& \leq I[H]-A_{0}^{2} \varepsilon^{2 \sqrt{2} \eta} .
\end{aligned}
$$

Since $\eta<1 / 8 \sqrt{2}$, we obtain

$$
\mu_{\varepsilon}\left(\mathbf{t}^{\varepsilon}\right) \leq N I[H]-A_{0}^{2} \varepsilon^{2 \sqrt{2} \eta} .
$$

In the second case, there holds

$$
M_{\varepsilon}\left(\mathbf{t}^{\varepsilon}\right) \leq I[H] \sum_{j=1}^{N} t_{j}^{n-1}-\left(\sqrt{2} A_{0}^{2}+o(1)\right) \varepsilon^{\sqrt{2} \eta} t_{j}^{n-1} \leq N I[H]-A_{0}^{2} \varepsilon^{\sqrt{2} \eta} .
$$

In the latter case, we have $t_{N}=1-\varepsilon(\log (1 / \varepsilon))^{2}$, and therefore

$$
\begin{aligned}
\mathcal{M}_{\varepsilon}\left(\mathbf{t}^{\varepsilon}\right) \leq I[H]\left(N-1+t_{N}^{n-1}\right) & +O(\varepsilon) \\
& \leq I[H]\left(N-(n-1) \varepsilon(\log (1 / \varepsilon))^{2}\right)+O(\varepsilon) .
\end{aligned}
$$

On the other hand, choosing $t_{j}=1-(j / \sqrt{2}) \varepsilon \log (1 / \varepsilon), j=1, \ldots, N$, we obtain

$$
\begin{aligned}
& \sum_{j=1}^{N} t_{j}^{n-1}=1-\frac{N(N+1)(n-1)}{2 \sqrt{2}} \varepsilon \log (1 / \varepsilon)+O\left(\varepsilon^{2}(\log (1 / \varepsilon))^{2}\right) ; \\
& e^{-2 \sqrt{2}\left(1-t_{1}\right) / \varepsilon}=\varepsilon^{2} ; \quad e^{-\sqrt{2}\left|t_{j-1}-t_{j}\right| / \varepsilon}=\varepsilon,
\end{aligned}
$$

and we find

$$
\mathcal{M}_{\varepsilon}\left(\mathbf{t}^{\varepsilon}\right) \geq N I[H]-\frac{N(N+1)(n-1)^{2}}{2 \sqrt{2}} \varepsilon \log (1 / \varepsilon)+O(\varepsilon),
$$

which contradicts either (5-2) or (5-3) or (5-4). This completes the proof of Proposition 5.1.

Remark 5.2. The above argument also shows that

$$
1-t_{1}^{\varepsilon} \sim \varepsilon \log (1 / \varepsilon), \quad t_{j-1}^{\varepsilon}-t_{j}^{\varepsilon} \sim \varepsilon \log (1 / \varepsilon) .
$$

Finally, we are ready to prove Theorem 1.1.

Proof of Theorem 1.1. By Proposition 3.2, there exists $\varepsilon_{N}$ such that, for $\varepsilon<\varepsilon_{N}$, we have a $C^{1}$ map $\mathbf{t} \mapsto \phi_{\varepsilon, \mathbf{t}}$ from $\bar{\Lambda}$ into $C^{2}\left(I_{\varepsilon}\right)$ such that

$$
\mathscr{Y}_{\varepsilon}\left[H_{\varepsilon, \mathbf{t}}+\phi_{\varepsilon, \mathbf{t}}\right]=\sum_{j=1}^{N} c_{j} Z_{\varepsilon, t_{j}}
$$

for some constants $\left\{c_{j}\right\} \subseteq \mathbb{R}$, which are also of class $C^{1}$ in $\mathbf{t}$. 
By Proposition 5.1, there exists $\mathbf{t}^{\varepsilon} \in \Lambda$ that achieves the maximum of $\mathscr{K}_{\varepsilon}: \mathbf{t} \mapsto$ $\mathscr{E}_{\varepsilon}\left[H_{\varepsilon, \mathbf{t}}+\phi_{\varepsilon, \mathbf{t}}\right]$. Let

$$
u_{\varepsilon}=\sum_{i=1}^{N}(-1)^{i} H_{\varepsilon, t_{i}^{\varepsilon}}+\phi_{\varepsilon, \mathbf{t}^{\varepsilon}}=H_{\varepsilon, \mathbf{t}^{\varepsilon}}+\phi_{\varepsilon, \mathbf{t}^{\varepsilon}}
$$

Then we have

and hence

$$
\left.\partial_{t_{i}}\right|_{\mathbf{t}=\mathbf{t}^{\varepsilon}} \mathcal{M}_{\varepsilon}\left(\mathbf{t}^{\varepsilon}\right)=0, \quad i=1, \ldots, N,
$$

$\left.\int_{I_{\varepsilon}}\left(\nabla u_{\varepsilon} \nabla \partial_{t_{i}}\left(H_{\varepsilon, \mathbf{t}}+\phi_{\varepsilon, \mathbf{t}}\right)+u_{\varepsilon} \partial_{t_{i}}\left(H_{\varepsilon, \mathbf{t}}+\phi_{\varepsilon, \mathbf{t}}\right)-f\left(u_{\varepsilon}\right) \partial_{t_{i}}\left(H_{\varepsilon, \mathbf{t}}+\phi_{\varepsilon, \mathbf{t}}\right)\right)\right|_{\mathbf{t}=\mathbf{t}^{\varepsilon}} r^{n-1} d r=0$.

Therefore, by (5-7), we find

$$
\sum_{j=1}^{N} c_{j} \int_{I_{\varepsilon}}\left(Z_{\varepsilon, t_{j}} \partial_{t_{i}}\left(H_{\varepsilon, \mathbf{t}}+\phi_{\varepsilon, \mathbf{t}}\right)\right) r^{n-1} d r=0
$$

Differentiating the equation $\left\langle\phi, Z_{\varepsilon, t_{j}}\right\rangle_{\varepsilon}=0$ with respect to $t_{j}$, we get

$$
\left\langle\partial_{t_{i}} \phi, Z_{\varepsilon, t_{j}}\right\rangle_{\varepsilon}=-\left\langle\phi, \partial_{t_{i}} Z_{\varepsilon, t_{j}}\right\rangle_{\varepsilon}=O\left(\|\phi\|_{*}\right) \varepsilon^{-n-1} .
$$

Using (3-3), we see that (5-8) is diagonally dominant in the coefficients $\left\{c_{i}\right\}$, which implies that $c_{j}=0$ for $j=1, \ldots, N$. Hence

$$
u_{\varepsilon}=H_{\varepsilon, \mathbf{t}^{\varepsilon}}+\phi_{\varepsilon, \mathbf{t}^{\varepsilon}}
$$

is a solution of (1-1).

By our construction, one can easily check that $\varepsilon^{n-1 \mathscr{E}_{\varepsilon}}\left(u_{\varepsilon}\right) \rightarrow N I[H]$ as $\varepsilon \rightarrow 0$, and that $u_{\varepsilon}$ has only $N$ zeroes $s_{1}^{\varepsilon} / \varepsilon, \ldots, s_{N}^{\varepsilon} / \varepsilon$. By the structure of $u_{\varepsilon}$, we see that (up to a permutation) $s_{i}^{\varepsilon}-t_{i}^{\varepsilon}=o(1)$. This proves (1) and (2) of Theorem 1.1.

It remains to prove (3). First we note that $u_{\varepsilon}^{\prime}$ satisfies

$$
\Delta u_{\varepsilon}^{\prime}+f^{\prime}\left(u_{\varepsilon}\right) u_{\varepsilon}^{\prime}=\frac{n-1}{r^{2}} u_{\varepsilon}^{\prime} .
$$

By our construction, at each interval $\left(s_{j}^{\varepsilon} / \varepsilon, s_{j-1}^{\varepsilon} / \varepsilon\right)$, for $j=2, \ldots, N$, there exists a point $\tilde{s}_{j-1}^{\varepsilon} / \varepsilon \in\left(s_{j} / \varepsilon, s_{j-1} / \varepsilon\right)$ such that $u_{\varepsilon}^{\prime}\left(\tilde{s}_{j-1}^{\varepsilon} / \varepsilon\right)=0$. Now, we set

$$
\begin{aligned}
\varphi_{1}(r) & = \begin{cases}u_{\varepsilon}^{\prime}(r) & \text { for } r \in\left(\tilde{s}_{1}^{\varepsilon} / \varepsilon, 1\right), \\
0 & \text { otherwise; }\end{cases} \\
\varphi_{j}(r) & = \begin{cases}u_{\varepsilon}^{\prime}(r) & \text { for } r \in\left(\tilde{s}_{j}^{\varepsilon} / \varepsilon, \tilde{s}_{j-1}^{\varepsilon} / \varepsilon\right), \\
0 & \text { otherwise, }\end{cases} \\
\varphi_{N}(r) & = \begin{cases}u_{\varepsilon}^{\prime}(r), & \text { for } r \in\left(1 /(2 \varepsilon), \tilde{s}_{N-1}^{\varepsilon} / \varepsilon\right), \\
2 \varepsilon(r-1 /(4 \varepsilon)) u_{\varepsilon}^{\prime}(r), & \text { for } 1 /(4 \varepsilon) \leq r \leq 1 /(2 \varepsilon), \\
0, & \text { for } r<1 /(4 \varepsilon) \text { or } r \geq \tilde{s}_{N-1}^{\varepsilon} / \varepsilon .\end{cases}
\end{aligned}
$$


Next, we define a quadratic functional

$$
\mathbf{Q}[\phi]=\int_{I_{\varepsilon}}\left(|\nabla \phi|^{2}-f^{\prime}\left(u_{\varepsilon}\right) \phi^{2}\right) r^{n-1} d r .
$$

It is easy to check that

$$
\int_{I_{\varepsilon}} \varphi_{i} \varphi_{j} r^{n-1} d r=0 \quad \text { for } i \neq j
$$

Using equation (5-9), we obtain

$$
\mathbf{Q}\left[\varphi_{i}\right]=-\int_{I_{\varepsilon}} \varphi_{i}^{2} r^{n-3} d r<0, \quad i=1, \ldots, N-1 .
$$

When $i=N$, we have

$$
\mathbf{Q}\left[\varphi_{N}\right]=-\int_{I_{\varepsilon}} \varphi_{N}^{2} r^{n-3} d r+O\left(e^{-1 /(C \varepsilon)}\right)<0 .
$$

From (5-12) and (5-13), the Morse index of $u_{\varepsilon}$ in $H_{r}^{1}\left(\Omega_{\varepsilon}\right)$ is at least $N$.

Finally, we also show that the Morse index of $u_{\varepsilon}$ in $H_{r}^{1}\left(\Omega_{\varepsilon}\right)$ is at most $N$. In fact, we define

$$
z_{j}^{\varepsilon}(r)=H_{\varepsilon, t_{j}^{\varepsilon}}^{\prime} \chi\left(\frac{\varepsilon r-t_{j}^{\varepsilon}}{\varepsilon \sqrt{|\log (1 / \varepsilon)|}}\right), \quad j=1, \ldots, N,
$$

and consider the following minimization problem

$$
\mu_{j}^{\varepsilon}=\inf _{\substack{\phi \in H^{1}\left(I_{\varepsilon, j}\right) \\ \int_{I_{\varepsilon, j}} \phi z_{j}^{\varepsilon} r^{n-1} d r=0}} \frac{\int_{I_{\varepsilon, j}}\left(|\nabla \phi|^{2}-f^{\prime}\left(u_{\varepsilon}\right) \phi^{2}\right) r^{n-1} d r}{\int_{I_{\varepsilon, j}} \phi^{2} r^{n-1} d r} .
$$

Assume that $\mu_{j}^{\varepsilon} \leq 0$. By standard regularity theory, $\mu_{j}^{\varepsilon}$ is attained by a function $\phi_{j}^{\varepsilon}$ which satisfies

$$
\begin{aligned}
& \Delta \phi_{j}^{\varepsilon}+f^{\prime}\left(u_{\varepsilon}\right) \phi_{j}^{\varepsilon}=-\mu_{j}^{\varepsilon} \phi_{j}^{\varepsilon}+c_{j}^{\varepsilon} z_{j}^{\varepsilon}, \\
& \left.\left(\phi_{j}^{\varepsilon}\right)^{\prime}\right|_{\partial I_{\varepsilon, j}}=0 \quad \text { and } \quad \int_{I_{\varepsilon, j}} \phi_{j}^{\varepsilon} z_{j} r^{n-1} d r=0,
\end{aligned}
$$

where $c_{j}^{\varepsilon}$ is a constant.

First, we notice that $c_{j}^{\varepsilon}=o\left(\left\|\phi_{j}^{\varepsilon}\right\|_{*}\right)$, which follows by reasoning as for (3-10) of Proposition 3.1. Then, from Lemma 2.1 we deduce that $\mu_{j}^{\varepsilon} \rightarrow 0$; moreover, the same argument leading to Proposition 3.1 shows that $\phi_{j}^{\varepsilon}=0$. 
Thus, $\mu_{j}^{\varepsilon}>0$. Let $\phi=\phi(r)$ be such that $\int_{I_{\varepsilon}} \phi z_{j}^{\varepsilon} r^{n-1}=0, j=1, \ldots, N$, which is equivalent to $\int_{I_{\varepsilon, j}} \phi z_{j}^{\varepsilon} r^{n-1}=0$. This then implies that

$$
\int_{I_{\varepsilon, j}}\left(|\nabla \phi|^{2}-f^{\prime}\left(u_{\varepsilon}\right) \phi^{2}\right) r^{n-1} d r \geq \mu_{j}^{\varepsilon} \int_{I_{\varepsilon, j}}|\phi|^{2} r^{n-1} d r, \quad j=1, \ldots, N
$$

and hence

$$
\begin{aligned}
\int_{I_{\varepsilon}}\left(|\nabla \phi|^{2}-f^{\prime}\left(u_{\varepsilon}\right) \phi^{2}\right) r^{n-1} d r=\sum_{j=1}^{N} \int_{I_{\varepsilon, j}}\left(|\nabla \phi|^{2}-f^{\prime}\left(u_{\varepsilon}\right) \phi^{2}\right) r^{n-1} d r \\
\geq \min _{j=1, \ldots, N} \mu_{j}^{\varepsilon} \int_{I_{\varepsilon}}|\phi|^{2} r^{n-1} d r
\end{aligned}
$$

This yields

$$
\lambda_{N+1}=\sup _{v_{1}, \ldots, v_{N}} \inf _{\int_{I_{\varepsilon}} \phi v_{j} r^{n-1}=0} \frac{\int_{I_{\varepsilon}}\left(|\nabla u|^{2}-f^{\prime}\left(u_{\varepsilon}\right) \phi^{2}\right) r^{n-1}}{\int_{I_{\varepsilon}} \phi^{2} r^{n-1}} \geq \min _{j=1, \ldots, N} \mu_{j}^{\varepsilon}>0
$$

and hence the Morse index of $u_{\varepsilon}$ in $H_{r}^{1}\left(\Omega_{\varepsilon}\right)$ is at most $N$.

Combining the upper and lower bound for the Morse index, we see that the Morse index of $u_{\varepsilon}$ in $H_{r}^{1}\left(\Omega_{\varepsilon}\right)$ is exactly $N$. This proves (3) of Theorem 1.1.

\section{Appendix}

In this appendix we expand the quantity $\mathscr{E}_{\varepsilon}\left[\sum_{j=1}^{N}(-1)^{j} H_{\varepsilon, t_{j}}\right]$ as a function of $\varepsilon$ and $\mathbf{t}$. Several facts will be used repeatedly:

$$
\begin{aligned}
& H(y)=1-A_{0} e^{-\sqrt{2}|y|}+O\left(e^{-2 \sqrt{2}|y|}\right), \text { for } y>1 ; \\
& H(y)=-1+A_{0} e^{-\sqrt{2}|y|}+O\left(e^{-2 \sqrt{2}|y|}\right), \text { for } y<-1 ; \\
& H^{\prime}(y)=\sqrt{2} A_{0} e^{-\sqrt{2}|y|}+O\left(e^{-2 \sqrt{2}|y|}\right), \text { for }|y|>1 ; \\
& \rho_{\varepsilon}\left(t_{1}\right)=\sqrt{2}\left(A_{0}+o(1)\right) e^{-\sqrt{2}\left(1-t_{1}\right) / \varepsilon} ; \\
& \rho_{\varepsilon}\left(t_{j}\right)=o\left(\rho_{\varepsilon}\left(t_{1}\right)\right) \text { for } j \geq 2 .
\end{aligned}
$$

From a Taylor expansion we find

$$
\mathscr{\mathscr { E }}_{\varepsilon}\left[H_{\varepsilon, \mathbf{t}}\right]=I_{1}+I_{2}+I_{3}+O\left(\varepsilon^{1-n} \rho_{\varepsilon}^{3}\left(t_{1}\right)\right)
$$


where

$$
\begin{aligned}
& I_{1}=\mathscr{E}_{\varepsilon}\left[\sum_{j=1}^{N}(-1)^{j} H_{t_{j}}\right], \\
& I_{2}=-\left(\sum_{l=1}^{K}(-1)^{l} \rho_{\varepsilon}\left(t_{l}\right)\right) \int_{I_{\varepsilon}}\left(\left(\sum_{j=1}^{N}(-1)^{j} H_{t_{j}}\right)^{\prime} \beta_{\varepsilon}^{\prime}-f\left(\sum_{j=1}^{N}(-1)^{j} H_{t_{j}}\right) \beta_{\varepsilon}\right) r^{n-1} d r, \\
& I_{3}=\frac{1}{2}\left(\sum_{l=1}^{N}(-1)^{l} \rho_{\varepsilon}\left(t_{l}\right)\right)^{2} \int_{I_{\varepsilon}}\left(\left|\beta_{\varepsilon}^{\prime}\right|^{2}-f^{\prime}\left(\sum_{j=1}^{N}(-1)^{j} H_{t_{j}}\right) \beta_{\varepsilon}^{2}\right) r^{n-1} .
\end{aligned}
$$

Recalling that $f^{\prime}( \pm 1)=-2$, the term $I_{3}$ can be estimated by

$$
\begin{aligned}
I_{3} & =\frac{1}{2}\left(\sum_{j=1}^{N}(-1)^{j} \rho_{\varepsilon}\left(t_{j}\right)\right)^{2} \int_{I_{\varepsilon}}\left(2-f^{\prime}\left(\sum_{j=1}^{N}(-1)^{j} H_{t_{j}}\right)\right) \beta_{\varepsilon}^{2} r^{n-1} d r+o\left(\varepsilon^{1-n} \rho_{\varepsilon}^{2}\left(t_{1}\right)\right) \\
& =\left(\rho_{\varepsilon}\left(t_{1}\right)\right)^{2} \int_{I_{\varepsilon}} \beta_{\varepsilon}^{2} r^{n-1} d r+o\left(\varepsilon^{1-n} \rho_{\varepsilon}^{2}\left(t_{1}\right)\right)=\frac{1}{2 \sqrt{2}} \varepsilon^{1-n}\left(\rho_{\varepsilon}\left(t_{1}\right)\right)^{2}+o\left(\varepsilon^{1-n} \rho_{\varepsilon}^{2}\left(t_{1}\right)\right) \\
& =\frac{A_{0}^{2}+o(1)}{\sqrt{2}} \varepsilon^{1-n} e^{-2 \sqrt{2}\left(1-t_{1}\right) / \varepsilon} .
\end{aligned}
$$

Next we estimate the integral in $I_{2}$. We have

$$
\begin{aligned}
\int_{I_{\varepsilon}}\left(\sum_{j=1}^{N}(\right. & \left.-1)^{j} H_{t_{j}}^{\prime} \beta_{\varepsilon}^{\prime}-f\left(\sum_{j=1}^{N}(-1)^{j} H_{t_{j}}\right) \beta_{\varepsilon}\right) r^{n-1} d r \\
& =\int_{I_{\varepsilon}}\left(\sqrt{2} \sum_{j=1}^{N}(-1)^{j} H_{t_{j}}^{\prime}-f\left(\sum_{j=1}^{N}(-1)^{j} H_{t_{j}}\right)\right) \beta_{\varepsilon} r^{n-1} d r \\
& =\int_{I_{\varepsilon, 1}}\left(-\sqrt{2} H_{t_{1}}^{\prime}-f\left(-H_{t_{1}}\right)\right) \beta_{\varepsilon} r^{n-1} d r+o\left(\varepsilon^{1-n} \rho_{\varepsilon}\left(t_{1}\right)\right) \\
& =-\frac{1}{\sqrt{2}} e^{-\sqrt{2}\left(1-t_{1}\right) / \varepsilon} \int_{\mathbb{R}}\left(\sqrt{2} H^{\prime}-f(H)\right) e^{\sqrt{2} y} d y\left(t_{1} / \varepsilon\right)^{n-1}+o\left(\varepsilon^{1-n} \rho_{\varepsilon}\left(t_{1}\right)\right) \\
& =-A_{0} e^{-\sqrt{2}\left(1-t_{1}\right) / \varepsilon}\left(t_{1} / \varepsilon\right)^{n-1}+o\left(\varepsilon^{1-n} \rho_{\varepsilon}\left(t_{1}\right)\right),
\end{aligned}
$$

since

Thus,

$$
\int_{\mathbb{R}}\left(\sqrt{2} H^{\prime}-f(H)\right) e^{\sqrt{2} y} d y=\left.\left(H^{\prime} e^{\sqrt{2} y}\right)\right|_{-\infty} ^{+\infty}=\sqrt{2} A_{0} .
$$

$$
I_{2}=-\left(\sqrt{2} A_{0}^{2}+o(1)\right) e^{-2 \sqrt{2}\left(1-t_{1}\right) / \varepsilon}\left(t_{1} / \varepsilon\right)^{n-1}+o\left(\varepsilon^{1-n} \rho_{\varepsilon}\left(t_{1}\right)\right)+O\left(\varepsilon^{2-n}\right),
$$

which implies that

(5-20) $I_{2}+I_{3}=-\frac{A_{0}^{2}+o(1)}{\sqrt{2}} e^{-2 \sqrt{2}\left(1-t_{1}\right) / \varepsilon}\left(t_{1} / \varepsilon\right)^{n-1}+o\left(\varepsilon^{1-n} \rho_{\varepsilon}\left(t_{1}\right)\right)+O\left(\varepsilon^{2-n}\right)$,

since $t_{1}=1+O\left(\varepsilon(\log (1 / \varepsilon))^{2}\right)$. 
It remains to consider $I_{1}$. For this purpose, we decompose it as

$$
I_{1}=\sum_{j=1}^{N} E_{\varepsilon, j}
$$

where

$$
\begin{aligned}
E_{\varepsilon, j} & =\int_{I_{\varepsilon, j}}\left(\frac{1}{2}\left|\sum_{l=1}^{N}(-1)^{l} H_{t_{l}}^{\prime}\right|^{2}-F\left(\sum_{l=1}^{N}(-1)^{l} H_{t_{l}}\right)\right) r^{n-1} d r \\
& =\int_{I_{\varepsilon, j}}\left(\frac{1}{2}\left|H_{t_{j}}^{\prime}+\sum_{l \neq j}(-1)^{j+l} H_{t_{l}}^{\prime}\right|^{2}-F\left(H_{j}+\sum_{l \neq j}(-1)^{j+l} H_{t_{l}}\right)\right) r^{n-1} d r \\
& =I_{4}+I_{5}+I_{6}+o\left(\varepsilon^{1-n} \sum_{i \neq j} e^{-\sqrt{2}\left|t_{i}-t_{j}\right| / \varepsilon}\right),
\end{aligned}
$$

with

$$
\begin{aligned}
& I_{4}=\int_{I_{\varepsilon, j}}\left(\frac{1}{2}\left|H_{t_{j}}^{\prime}\right|^{2}-F\left(H_{t_{j}}\right)\right) r^{n-1} d r, \\
& I_{5}=\int_{I_{\varepsilon, j}}\left(H_{t_{j}}^{\prime} \sum_{l \neq j}(-1)^{l+j} H_{t_{l}}^{\prime}-f\left(H_{t_{j}}\right) \sum_{l \neq j}(-1)^{l+j} H_{t_{l}}\right) r^{n-1} d r, \\
& I_{6}=\frac{1}{2} \int_{I_{\varepsilon, j}}\left|\sum_{l \neq j}(-1)^{j+l} H_{t_{l}}\right|^{2}\left(2-f^{\prime}\left((-1)^{j} H_{t_{j}}\right)\right) r^{n-1} d r .
\end{aligned}
$$

Using the fact that $\left|H^{\prime}\right|^{2}=2 F(H)$, for $I_{4}$ we find

$$
\begin{aligned}
I_{4} & =\int_{I_{\varepsilon, j}}\left|H_{t_{j}}^{\prime}\right|^{2} r^{n-1} d r \\
& =\int_{R}\left|H^{\prime}\right|^{2} d y\left(t_{j} / \varepsilon\right)^{n-1}-\frac{A_{0}^{2}+o(1)}{\sqrt{2}}\left(e^{-\sqrt{2}\left|t_{j}-t_{j-1}\right| / \varepsilon}+e^{-\sqrt{2}\left|t_{j}-t_{j+1}\right| / \varepsilon}\right)\left(t_{j} / \varepsilon\right)^{n-1} \\
& +O\left(\varepsilon^{2-n}\right) .
\end{aligned}
$$

For $j \geq 2, \quad I_{5}$ can be estimated (by recalling the exponential-decay property of $H(y) \pm 1)$ as

$$
\begin{aligned}
I_{5} & =\left.\left(t_{j} / \varepsilon\right)^{n-1} H_{t_{j}}^{\prime} \sum_{l \neq j}(-1)^{l+j} H_{t_{l}}\right|_{\partial I_{\varepsilon, j}}+O\left(\varepsilon^{2-n}\right) \\
& =-\left(A_{0}^{2}+o(1)\right) \sqrt{2}\left(e^{-\sqrt{2}\left|t_{j}-t_{j-1}\right| / \varepsilon}+e^{-\sqrt{2}\left|t_{j}-t_{j+1}\right| / \varepsilon}\right)\left(t_{j} / \varepsilon\right)^{n-1}+O\left(\varepsilon^{2-n}\right) .
\end{aligned}
$$

For $j=1$, we have

$$
\begin{aligned}
I_{5} & =\left.\left(t_{1} / \varepsilon\right)^{n-1} H_{t_{j}}^{\prime} \sum_{l>1}(-1)^{l+1} H_{t_{l}}\right|_{\partial I_{\varepsilon, 1}}+O\left(\varepsilon^{2-n}\right) \\
& =-\left(A_{0}^{2}+o(1)\right) \sqrt{2} e^{-\sqrt{2}\left|t_{1}-t_{2}\right| / \varepsilon}\left(t_{j} / \varepsilon\right)^{n-1}+O\left(\varepsilon^{2-n}\right) .
\end{aligned}
$$


$I_{6}$ can be estimated similarly: for $j \geq 2$, we have

$$
\begin{aligned}
I_{6} & =2 \int_{I_{\varepsilon, j}}\left|\sum_{l \neq j}(-1)^{j+l} H_{t_{l}}\right|^{2} r^{n-1} d r \\
& =\frac{A_{0}^{2}+o(1)}{\sqrt{2}}\left(e^{-\sqrt{2}\left|t_{j}-t_{j-1}\right| / \varepsilon}+e^{-\sqrt{2}\left|t_{j}-t_{j+1}\right| / \varepsilon}\right)\left(t_{j} / \varepsilon\right)^{n-1}+O\left(\varepsilon^{2-n}\right),
\end{aligned}
$$

while for $j=1$,

$I_{6}=2 \int_{I_{\varepsilon, 1}}\left|\sum_{l>1}(-1)^{l+1} H_{t_{l}}\right|^{2} r^{n-1} d r=\frac{A_{0}^{2}+o(1)}{\sqrt{2}} e^{-\sqrt{2}\left|t_{1}-t_{2}\right| / \varepsilon}\left(t_{1} / \varepsilon\right)^{n-1}+O\left(\varepsilon^{2-n}\right)$.

Combining the estimates of $I_{4}, I_{5}$, and $I_{6}$, we obtain

$$
\begin{gathered}
I_{1}=I[H] \sum_{j=1}^{N}\left(t_{j} / \varepsilon\right)^{n-1}-\sqrt{2}\left(A_{0}^{2}+o(1)\right) \sum_{j=2}^{N} e^{-\sqrt{2}\left|t_{j}-t_{j-1}\right| / \varepsilon}\left(t_{j} / \varepsilon\right)^{n-1} \\
-\frac{A_{0}^{2}+o(1)}{\sqrt{2}} e^{-2 \sqrt{2}\left(1-t_{1}\right) / \varepsilon}+O\left(\varepsilon^{2-n}\right) \\
=I[H] \sum_{j=1}^{N}\left(t_{j} / \varepsilon\right)^{n-1}-\sqrt{2}\left(A_{0}^{2}+o(1)\right) \sum_{j=2}^{N} e^{-\sqrt{2}\left|t_{j}-t_{j-1}\right| / \varepsilon}\left(t_{j} / \varepsilon\right)^{n-1} \\
-\frac{A_{0}^{2}+o(1)}{\sqrt{2}} e^{-2 \sqrt{2}\left(1-t_{1}\right) / \varepsilon}\left(t_{1} / \varepsilon\right)^{n-1}+O\left(\varepsilon^{2-n}\right) .
\end{gathered}
$$

Adding this to the estimate in (5-20), we obtain the asymptotic expansion (2-20) of $\mathscr{E}_{\varepsilon}\left[\sum_{j=1}^{N}(-1)^{j} H_{\varepsilon, t_{j}}\right]$.

\section{References}

[Alikakos et al. 2000] N. D. Alikakos, X. Chen, and G. Fusco, "Motion of a droplet by surface tension along the boundary", Calculus of Variations and Partial Differential Equations 11:3 (2000), 233-305. MR 2001k:35016 Zbl 0994.35052

[Allen and Cahn 1979] S. Allen and J. W. Cahn, "A microscopic theory for antiphase boundary motion and its application to antiphase domain coarsening", Acta. Metall. 27 (1979), 1084-1095.

[Ambrosetti et al. 2003] A. Ambrosetti, A. Malchiodi, and W.-M. Ni, "Singularly perturbed elliptic equations with symmetry: existence of solutions concentrating on spheres, I", Comm. Math. Phys. 235:3 (2003), 427-466. MR 2004c:35014 Zbl 1072.35019

[Ambrosetti et al. 2004] A. Ambrosetti, A. Malchiodi, and W.-M. Ni, "Singularly perturbed elliptic equations with symmetry: existence of solutions concentrating on spheres, II", Indiana Univ. Math. J. 53:2 (2004), 297-329. MR 2005c:35015 Zbl 1081.35008

[Bronsard and Stoth 1996] L. Bronsard and B. Stoth, "On the existence of high multiplicity interfaces”, Math. Res. Lett. 3:1 (1996), 41-50. MR 97d:35199 Zbl 0853.35008

[Dancer and Yan 1999] E. N. Dancer and S. Yan, "Multipeak solutions for a singularly perturbed Neumann problem”, Pacific J. Math. 189:2 (1999), 241-262. MR 2000d:35010 Zbl 0933.35070 
[Dancer and Yan 2003] E. N. Dancer and S. Yan, "Multi-layer solutions for an elliptic problem", $J$. Differential Equations 194:2 (2003), 382-405. MR 2004i:35098 Zbl 02005884

[Flores et al. 2001] G. Flores, P. Padilla, and Y. Tonegawa, "Higher energy solutions in the theory of phase transitions: a variational approach", J. Differential Equations 169:1 (2001), 190-207. MR 2001m:35089 Zbl 0970.35037

[Gui and Wei 1999] C. Gui and J. Wei, "Multiple interior peak solutions for some singularly perturbed Neumann problems", Journal of Diff. Equations 158:1 (1999), 1-27. MR 2000g:35035 Zbl 1061.35502

[Gui and Wei 2000] C. Gui and J. Wei, "On multiple mixed interior and boundary peak solutions for some singularly perturbed Neumann problems", Canad. J. Math. 52:3 (2000), 522-538. MR 2001b:35023 Zbl 0949.35052

[Gui et al. 2000] C. Gui, J. Wei, and M. Winter, "Multiple boundary peak solutions for some singularly perturbed Neumann problems", Ann. Inst. H. Poincaré Anal. Non Linéaire 17:1 (2000), 47-82. MR 2001a:35018 Zbl 0944.35020

[Kohn and Sternberg 1989] R. V. Kohn and P. Sternberg, "Local minimisers and singular perturbations”, Proc. Roy. Soc. Edinburgh Sect. A 111:1-2 (1989), 69-84. MR 90c:49021 Zbl 0676.49011

[Kowalczyk 2005] M. Kowalczyk, "On the existence and Morse index of solutions to the AllenCahn equation in two dimensions", Ann. Mat. Pura Appl. (4) 184:1 (2005), 17-52. MR 2006f:35088

[Malchiodi et al. 2005] A. Malchiodi, W.-M. Ni, and J. Wei, "Multiple clustered layer solutions for semilinear Neumann problems on a ball", Ann. Inst. H. Poincaré Anal. Non Linéaire 22:2 (2005), 143-163. MR 2006i:35098 Zbl 02165096

[Modica 1987] L. Modica, "The gradient theory of phase transitions and the minimal interface criterion”, Arch. Rational Mech. Anal. 98:2 (1987), 123-142. MR 88f:76038 Zbl 0616.76004

[Müller 1993] S. Müller, "Singular perturbations as a selection criterion for periodic minimizing sequences”, Calculus of Var. and Partial Differential Equations 1:2 (1993), 169-204. MR 95k:49030 Zbl 0821.49015

[Nakashima 2003] K. Nakashima, "Multi-layered stationary solutions for a spatially inhomogeneous Allen-Cahn equation”, J. Differential Equations 191:1 (2003), 234-276. MR 2004f:35097 Zbl 1034.34024

[Nakashima and Tanaka 2003] K. Nakashima and K. Tanaka, "Clustering layers and boundary layers in spatially inhomogeneous phase transition problems", Ann. Inst. H. Poincaré Anal. Non Linéaire 20:1 (2003), 107-143. MR 2004b:35016 Zbl 01901029

[Padilla and Tonegawa 1998] P. Padilla and Y. Tonegawa, "On the convergence of stable phase transitions”, Comm. Pure Appl. Math. 51:6 (1998), 551-579. MR 99b:58054 Zbl 0955.58011

[Rabinowitz and Stredulinsky 2003] P. H. Rabinowitz and E. Stredulinsky, "Mixed states for an Allen-Cahn type equation”, Comm. Pure Appl. Math. 56:8 (2003), 1078-1134. Dedicated to the memory of Jürgen K. Moser. MR 2004i:35125 Zbl 01981618

[Rabinowitz and Stredulinsky 2004] P. H. Rabinowitz and E. Stredulinsky, "Mixed states for an Allen-Cahn type equation. II", Calc. Var. Partial Differential Equations 21:2 (2004), 157-207. MR 2005e:35080 Zbl 02113881

[Sternberg and Zumbrun 1998] P. Sternberg and K. Zumbrun, "Connectivity of phase boundaries in strictly convex domains”, Arch. Rational Mech. Anal. 141:4 (1998), 375-400. MR 99c:49045 Zbl 0911.49025

Received March 21, 2005. 


\section{ANDREA MALCHIODI}

SECtor of FunCtional AnALysis And ApPlications, SiSSA

VIA BEIRUT 2-4

34014 TRIESTE

ITALY

malchiod@sissa.it

\section{WEI-Ming Ni}

SCHOOL OF MATHEMATICS

UNIVERSITY OF MINNESOTA

MINNEAPOLIS, MN 55455

UNITED STATES

ni@math.umn.edu

www.math.umn.edu

JUNCHENG WEI

DEPARTMENT OF MATHEMATICS

THE Chinese University OF HONG KONG

SHATIN

HONG KONG

wei@math.cuhk.edu.hk

www.math.cuhk.edu.hk 